February 5, 1996

hep-th/

UMN-TH-1421/96

NUC-MINN-96/2-T

\title{
Confinement and Chiral Condensates in 2-d QED with Massive N-flavor Fermions
}

\author{
Ramón Rodríguez ${ }^{1}$ and Yutaka Hosotani ${ }^{2}$ \\ School of Physics and Astronomy, University of Minnesota \\ Minneapolis, Minnesota 55455, U.S.A. \\ ${ }^{1}$ rodriguez@physics.spa.umn.edu \\ ${ }^{2}$ yutaka@mnhep.hep.umn.edu
}

\begin{abstract}
We evaluate Polyakov loops and string tension in two-dimensional QED with both massless and massive $N$-flavor fermions at zero and finite temperature. External charges, or external electric fields, induce phases in fermion masses and shift the value of the vacuum angle parameter $\theta$, which in turn alters the chiral condensate. In particular, in the presence of two sources of opposite charges, $q$ and $-q$, the shift in $\theta$ is $2 \pi(q / e)$ independent of $N$. The string tension has a cusp singularity at $\theta= \pm \pi$ for $N \geq 2$ and is proportional to $m^{2 N /(N+1)}$ at $T=0$.
\end{abstract}

Two-dimensional QED, the Schwinger model, with massive $N$-flavor fermions resembles fourdimensional QCD in various aspects, including confinement, chiral condensates, and $\theta$ vacua [1]- 11]. Much progress has been made recently in evaluating chiral condensates and string tension in the massive theory [12]-[16]. In this paper we shall show that the three phenomena, confinement, chiral condensates, and $\theta$ vacua, are intimately related to each other. In particular, the string tension in the confining potential is determined by the $\theta$ dependence of chiral condensates $\langle\bar{\psi} \psi\rangle$.

The behavior of the model is distinctively different, depending on whether $N=1$ (one-flavor) or $N \geq 2$ (multi-flavor), and on whether fermions are massless or massive. The massless $(m=0)$ theory is exactly solvable. $\langle\bar{\psi} \psi\rangle_{\theta} \neq 0$ for $N=1$, but $\langle\bar{\psi} \psi\rangle_{\theta}=0$ for $N \geq 2$. [17, 18] In either cases the string tension between two external sources of opposite charge vanishes [4, 8, 12].

In the massive $(m \neq 0)$ theory $\langle\bar{\psi} \psi\rangle_{\theta}$ is proportional to $m^{(N-1) /(N+1)} \cos ^{2 N /(N+1)}(\bar{\theta} / N)$ at $T=0$ where $\bar{\theta}=\theta-2 \pi[(\theta+\pi) / 2 \pi]$ [5, 13]. For $N \geq 2$ the dependence on $m$ is non-analytic. It also has a cusp singularity at $\theta= \pm \pi$. A perturbation theory in fermion masses is not valid at low temperature. 
The confinement phenomenon can be explored in various ways. One way is to evaluate the Polyakov loop $P_{q}(x)=\exp \left\{i q \int_{0}^{\beta} d \tau A_{0}(\tau, x)\right\}$ at finite temperature $T=\beta^{-1}$ [19]-23. $F(T)=$ $-T \ln \left\langle P_{q}(x)\right\rangle$ or $-T \ln \left\langle P_{q}(x)^{\dagger} P_{q}(y)\right\rangle$ measures the increase in free energy in the presence of an external charge $q$ or a pair of charges $q$ and $-q$. In particular, the latter is written as $\sigma|x-y|$ for large $|x-y|$ where $\sigma$ is identified with the string tension. This method has the advantage of giving the temperature dependence directly.

Alternatively one may determine the ground state to evaluate the change in the energy density (at $T=0$ ) when a pair of sources of charge $q$ and $-q$ is placed $4,24,25$. This method has the advantage of showing how external charges affect the $\theta$ parameter and chiral condensates.

We employ both methods in a unified manner. Years ago Coleman, Jackiw, and Susskind showed the confinement of fractional charges in the $N=1$ theory adopting the latter method 溲. Recently Hansson, Nielsen and Zahed applied functional integration method to evaluate the Polyakov loop correlation function [12]. The argument has been generalized to finite temperature by Grignani et al. [15]. Ellis et al. [8] and Gross et al. [14] have presented the mechanism of confinement in terms of soliton solutions in the bosonized form. All of these arguments are given in the one flavor $(N=1)$ case and rely on the validity of a perturbation theory in a fermion mass.

Recently chiral condensates with arbitrary fermion masses $m$, vacuum angle $\theta$ and temperature $T$ have been evaluated in the $N$-flavor model [13]. The problem was reduced to solving a quantum mechanical system of $N$ degrees of freedom. It was shown that the $m \rightarrow 0$ and $T \rightarrow 0$ limits do not commute for $N \geq 2$. In particular, the $m$-dependence of physical quantities is singular at $T=0$.

We analyse the model

$$
\begin{aligned}
& \mathcal{L}=-\frac{1}{4} F_{\mu \nu} F^{\mu \nu}+\sum_{a=1}^{N} \bar{\psi}_{a}\left\{\gamma^{\mu}\left(i \partial_{\mu}-e A_{\mu}\right)\right\} \psi_{a}+\mathcal{L}_{\text {mass }} \\
& \mathcal{L}_{\text {mass }}=-\sum_{a=1}^{N} m_{a}\left\{e^{i \delta_{a}} M_{a}+e^{-i \delta_{a}} M_{a}^{\dagger}\right\} \quad\left(m_{a} \geq 0\right) \\
& M_{a}=\bar{\psi}_{a} \frac{1}{2}\left(1-\gamma^{5}\right) \psi_{a}
\end{aligned}
$$

defined on a circle with a circumference $L$ [24]-35]. The model defined at finite temperature 36][46], on a torus or sphere [47- 52], or on a lattice or light-cone [53] 62], has been also extensively discussed in the literature.

We impose boundary conditions $A_{\mu}(t, x+L)=A_{\mu}(t, x)$ and $\psi_{a}(t, x+L)=-\psi_{a}(t, x)$. On a circle the only physical degree of freedom associated with gauge fields is the Wilson line phase $\Theta_{\mathrm{W}}(t): 24$

$$
e^{i \Theta_{\mathrm{W}}(t)}=\exp \left\{i e \int_{0}^{L} d x A_{1}(t, x)\right\} .
$$

In Matusbara's formalism the finite temperature field theory is defined by imposing perodic or anti-periodic boundary conditions in the imaginary time $(\tau)$ axis on bosons or fermions, respectively. Mathematically the model at finite temperature $T=\beta^{-1}$ is obtained from the model defined on a circle by Wick rotation and replacement $L \rightarrow \beta$, it $\rightarrow x$ and $x \rightarrow \tau$. Furthermore the Polyakov 
loop of a charge $q$ in the finite temperature theory corresponds to the Wilson line phase:

$$
P_{q}(x)=\exp \left\{i q \int_{0}^{\beta} d \tau A_{0}(\tau, x)\right\} \Longleftrightarrow \exp \left\{i \frac{q}{e} \Theta_{\mathrm{W}}(t)\right\} .
$$

We bosonize fermions in the Coulomb gauge [5, 17, 24, 13]. Take $\gamma^{\mu}=\left(\sigma_{1}, i \sigma_{2}\right)$ and write $\psi_{a}^{T}=\left(\psi_{+}^{a}, \psi_{-}^{a}\right)$. In the interaction picture defined by free massless fermions

$$
\begin{gathered}
\psi_{ \pm}^{a}(t, x)=\frac{1}{\sqrt{L}} C_{ \pm}^{a} e^{ \pm i\left\{q_{ \pm}^{a}+2 \pi p_{ \pm}^{a}(t \pm x) / L\right\}}: e^{ \pm i \sqrt{4 \pi} \phi_{ \pm}^{a}(t, x)}: \\
\left.\left.e^{2 \pi i p_{ \pm}^{a}} \mid \text { phys }\right\rangle=\mid \text { phys }\right\rangle
\end{gathered}
$$

where $C_{+}^{a}=\exp \left\{i \pi \sum_{b=1}^{a-1}\left(p_{+}^{b}+p_{-}^{b}\right)\right\}$ and $C_{-}^{a}=\exp \left\{i \pi \sum_{b=1}^{a}\left(p_{+}^{b}-p_{-}^{b}\right)\right\}$. Here

$$
\begin{aligned}
{\left[q_{ \pm}^{a}, p_{ \pm}^{b}\right] } & =i \delta^{a b} \\
\phi_{ \pm}^{a}(t, x) & =\sum_{n=1}^{\infty}(4 \pi n)^{-1 / 2}\left\{c_{ \pm, n}^{a} e^{-2 \pi i n(t \pm x) / L}+\text { h.c. }\right\} \quad, \quad\left[c_{ \pm, n}^{a}, c_{ \pm, m}^{b, \dagger}\right]=\delta^{a b} \delta_{n m}
\end{aligned}
$$

The : : in (4) indicates normal ordering with respect to $\left(c_{n}, c_{n}^{\dagger}\right)$. In physical states $p_{ \pm}^{a}$ takes an integer eigenvalue.

Conjugate pairs are $\left\{p_{a}, q_{a}\right\}=\left\{\frac{1}{2}\left(p_{+}^{a}+p_{-}^{a}\right), q_{+}^{a}+q_{-}^{a}\right\},\left\{\tilde{p}_{a}, \tilde{q}_{a}\right\}=\left\{p_{+}^{a}-p_{-}^{a}, \frac{1}{2}\left(q_{+}^{a}-q_{-}^{a}\right)\right\}$, $\left\{P_{\mathrm{W}}, \Theta_{\mathrm{W}}\right\}$, and $\left\{\Pi_{a}, \phi_{a}=\phi_{+}^{a}+\phi_{-}^{a}\right\}$. The Hamiltonian in the Schrödinger picture becomes

$$
\begin{aligned}
& H_{\text {tot }}=H_{0}+H_{\phi}+H_{\text {mass }}+(\text { constant }) \\
& H_{0}=\frac{e^{2} L}{2} P_{\mathrm{W}}^{2}+\frac{N}{2 \pi L}\left\{\Theta_{\mathrm{W}}+\frac{2 \pi}{N} \sum_{a=1}^{N} p_{a}\right\}^{2}-\frac{2 \pi}{N L}\left\{\sum_{a=1}^{N} p_{a}\right\}^{2}+\frac{2 \pi}{L} \sum_{a=1}^{N}\left\{p_{a}^{2}+\frac{1}{4} \tilde{p}_{a}^{2}\right\} \\
& H_{\phi}=\int_{0}^{L} d x \frac{1}{2}:\left[\sum_{a=1}^{N}\left\{\Pi_{a}^{2}+\left(\phi_{a}^{\prime}\right)^{2}\right\}+\frac{e^{2}}{\pi}\left(\sum_{a=1}^{N} \phi_{a}\right)^{2}\right]: \\
& H_{\text {mass }}=\int_{0}^{L} d x \sum_{a} m_{a}\left\{e^{i \delta_{a}} M_{a}+e^{-i \delta_{a}} M_{a}^{\dagger}\right\} .
\end{aligned}
$$

In the mass term

$$
M_{a}=-C_{-}^{a \dagger} C_{+}^{a} \cdot e^{-2 \pi i \tilde{p}_{a} x / L} e^{i q_{a}} \cdot L^{-1} N_{0}\left[e^{i \sqrt{4 \pi} \phi_{a}}\right]
$$

where $N_{\mu}[\cdots]$ indicates that the operator inside [ ] is normal-ordered with respect to a mass $\mu$. In general a mass-eigenstate field $\chi_{\alpha}$ with a mass $\mu_{\alpha}$ is related to $\phi_{a}$ by an orthogonal transformation $\chi_{\alpha}=U_{\alpha a} \phi_{a}$. In (7) we have

$$
\begin{aligned}
& N_{0}\left[e^{i \sqrt{4 \pi} \phi_{a}}\right]=\bar{B}_{a} \prod_{\alpha=1}^{N} N_{\mu_{\alpha}}\left[e^{i U_{\alpha a} \sqrt{4 \pi} \chi_{\alpha}}\right] \\
& \bar{B}_{a}=\prod_{\alpha=1}^{N} B\left(\mu_{\alpha} L\right)^{\left(U_{\alpha a}\right)^{2}} \\
& B(z)=\frac{z}{4 \pi} \exp \left\{\gamma+\frac{\pi}{z}-2 \int_{1}^{\infty} \frac{d u}{\left(e^{u z}-1\right) \sqrt{u^{2}-1}}\right\} .
\end{aligned}
$$


As $\left[\tilde{p}_{a}, H_{\text {tot }}\right]=0$, we may restrict ourselves to states with $\tilde{p}_{a}=0$.

$H_{\text {mass }}$ gives rather complicated coupling between the zero and $\phi_{a}\left(\chi_{\alpha}\right)$ modes, whose effects are non-perturbative for $N \geq 2$. As in previous papers [13] the vacuum wave function is written in the form

$$
\left|\Phi_{\mathrm{vac}}(\theta)\right\rangle=\int_{0}^{2 \pi} \prod_{a=1}^{N-1} d \varphi_{a} f\left(\varphi_{a} ; \theta_{\mathrm{eff}}\right)\left|\Phi_{0}\left(\varphi_{a}+\delta_{a} ; \theta\right)\right\rangle
$$

where

$$
\begin{aligned}
& \left|\Phi_{0}\left(\varphi_{a} ; \theta\right)\right\rangle=(2 \pi)^{-N / 2} \sum_{\left\{n, r_{a}\right\}} e^{i n \theta+i \sum_{a=1}^{N-1} r_{a} \varphi_{a}}\left|\Phi_{0}^{\left(n+r_{1}, \cdots, n+r_{N-1}, n\right)}\right\rangle \\
& \left\langle\Theta_{\mathrm{W}}, q_{a} \mid \Phi_{0}^{\left(n_{1}, \cdots, n_{N}\right)}\right\rangle=u_{0}\left(\Theta_{\mathrm{W}}+\frac{2 \pi}{N} \sum_{a=1}^{N} n_{a}\right)(2 \pi)^{-N / 2} e^{i \sum_{a=1}^{N} n_{a} q_{a}} \\
& u_{0}(x)=\left(\frac{N}{\pi^{2} \mu L}\right)^{1 / 4} e^{-N x^{2} / 2 \pi \mu L} \quad, \quad \mu^{2}=\frac{N e^{2}}{\pi} \\
& \theta_{\mathrm{eff}}=\theta-\sum_{a=1}^{N} \delta_{a} .
\end{aligned}
$$

We have generalized the expression to incorporate the phases $\delta_{a}$ 's in the mass parameter in (1). The ansatz above for the vacuum is good for $m_{a} \ll e$.

The eigenvalue equation $\left(H_{0}+H_{\text {mass }}\right)\left|\Phi_{\text {vac }}(\theta)\right\rangle=E\left|\Phi_{\text {vac }}(\theta)\right\rangle$ reads

$$
\left\{-\triangle_{N}^{\varphi}+V_{N}\left(\varphi ; \theta_{\text {eff }}\right)\right\} f\left(\varphi ; \theta_{\text {eff }}\right)=\epsilon f\left(\varphi ; \theta_{\text {eff }}\right)
$$

where

$$
\begin{aligned}
\triangle_{N}^{\varphi} & =\sum_{a=1}^{N-1} \frac{\partial^{2}}{\partial \varphi_{a}^{2}}-\frac{2}{N-1} \sum_{a<b}^{N-1} \frac{\partial^{2}}{\partial \varphi_{a} \partial \varphi_{b}} \\
V_{N}\left(\varphi ; \theta_{\text {eff }}\right) & =-\frac{N L}{(N-1) \pi} e^{-\pi / N \mu L} \sum_{a=1}^{N} m_{a} \bar{B}_{a} \cos \varphi_{a} \\
\varphi_{N} & =\theta_{\text {eff }}-\sum_{a=1}^{N-1} \varphi_{a}
\end{aligned}
$$

and $\epsilon=N E L / 2 \pi(N-1)$.

For the $\chi_{\alpha}$ fields, the vacuum is defined with respect to their physical mass $\mu_{\alpha}$ 's which needs to be determined self-consistently from the wave function in (9). In the symmetric case $m_{a}=m$ one has $\mu_{2}=\cdots=\mu_{N}, \mu_{1}^{2}=\mu^{2}+\mu_{2}^{2}$ and $\bar{B}_{a}=B\left(\mu_{1} L\right)^{1 / N} B\left(\mu_{2} L\right)^{(N-1) / N} \equiv \bar{B}$. The potential is reduced to

$$
\begin{aligned}
& V_{N}\left(\varphi ; \theta_{\text {eff }}\right)=-\kappa_{0} \sum_{a=1}^{N} \cos \varphi_{a} \\
& \kappa_{0}=\frac{N m L}{(N-1) \pi} e^{-\pi / N \mu L} \bar{B} .
\end{aligned}
$$

Further $\mu_{2}^{2}$ is determined by

$$
\mu_{2}^{2}=R=\frac{8 \pi m \bar{B}}{L} e^{-\pi / N \mu L}\langle\cos \varphi\rangle_{f}
$$


where the $f$-average is given by $\langle g(\varphi)\rangle_{f}=\int[d \varphi] g(\varphi)|f(\varphi)|^{2}$. We have made use of the fact that $\left\langle e^{i \varphi_{a}}\right\rangle_{f}$ is independent of $a$. Eqs. (11) and (14) are solved simultaneously. Evaluation of these equations was given in 13]. An important point in the following discussion is that $f\left(\varphi ; \theta_{\text {eff }}\right)$ or $\Phi_{\mathrm{vac}}(\theta)$ is determined solely by $m, L$ (or $T$ ), and $\theta_{\text {eff. }}$.

Now let us evaluate the Polyakov loop at finite $T$, or equivalently $\left\langle e^{i k \Theta_{\mathrm{W}}(t)}\right\rangle_{\theta}$ on a circle. The parameter $k$ corresponds to $q / e$ where $q$ is the charge of an external source. Since the expectation value is $t$-translation invariant, it is sufficient to evaluate at $t=0$. Making use of (9), one immediately finds

$$
\left\langle\Phi\left(\theta^{\prime} ; \varphi^{\prime}\right)\left|e^{i k \Theta_{\mathrm{W}}}\right| \Phi(\theta ; \varphi)\right\rangle=\delta_{2 \pi}\left(\theta-\theta^{\prime}-2 \pi k\right) \prod_{a=1}^{N-1} \delta_{2 \pi}\left(\varphi_{a}-\varphi_{a}^{\prime}-\frac{2 \pi k}{N}\right) \cdot e^{-\pi k^{2} \mu L / 4 N} .
$$

It follows that

$$
\begin{aligned}
& \left\langle P_{k e}\right\rangle_{\theta, T}=\left\langle e^{i k \Theta_{\mathrm{W}}}\right\rangle_{\theta, L=T^{-1}} \\
& = \begin{cases}0 & \text { for } k \neq \text { an integer } \\
e^{-k^{2} \pi \mu / 4 N T} \int[d \varphi] f\left(\varphi_{a} ; \theta_{\mathrm{eff}}\right)^{*} f\left(\varphi_{a}+\frac{2 \pi k}{N} ; \theta_{\text {eff }}\right) & \text { for } k=\text { an integer } .\end{cases}
\end{aligned}
$$

The vanishing of the Polyakov loop for a fractional $k$ is due to the invariance under large gauge transformations. The Hamiltonian (6) is invariant under $\Theta_{\mathrm{W}} \rightarrow \Theta_{\mathrm{W}}+2 \pi$ and $p_{a} \rightarrow p_{a}-1$. In other words,

$$
\begin{aligned}
& U=\exp \left(2 \pi i P_{\mathrm{W}}+i \sum_{a=1}^{N} q_{a}\right) \\
& {\left[U, H_{\mathrm{tot}}\right]=0} \\
& U\left|\Phi_{\mathrm{vac}}(\theta)\right\rangle=e^{i \theta}\left|\Phi_{\mathrm{vac}}(\theta)\right\rangle,
\end{aligned}
$$

which implies the vanishing of $\left\langle e^{i k \Theta_{\mathrm{W}}}\right\rangle_{\theta}$ for a non-integer $k$.

In the $N=1$ (one-flavor) case, there is no $\varphi_{a}$ degrees of freedom. Eq. (16) reduces to $\left\langle P_{k e}\right\rangle_{\theta, T}=$ $e^{-k^{2} \pi \mu / 4 T}$, which agrees with the result of Grignani et al. The factor $k^{2} \pi \mu / 4$ is understood as the self-energy of the source [15].

In the $N \geq 2$ (multi-flavor) case the overlap integral for the $f\left(\varphi ; \theta_{\text {eff }}\right)$ factor becomes relevant. In the massless $(m=0)$ case, however, $f(\varphi)$ is constant as the potential $V_{N}\left(\varphi ; \theta_{\text {eff }}\right)$ in (11) vanishes. Hence $\left\langle P_{k e}\right\rangle_{\theta, T}=e^{-k^{2} \pi \mu / 4 N T}$ in the massless theory.

When $m \neq 0$, the overlap integral needs to be evaluated numerically. In two limits, namely $T / \mu \ll(m / \mu)^{\frac{N}{N+1}}$ and $T / \mu \gg 1$, analytic expressions are obtained. It is instructive to examine the free energy $F_{e}(T)=-T \ln \left\langle P_{e}\right\rangle_{\theta, T}$ for $k=1$. At sufficiently low $T=L^{-1}, \kappa_{0} \gg 1$ in (13) so that $f(\varphi)$ has a sharp peak at the minimum of the potential

$$
\varphi_{a}^{\mathrm{min}}=\frac{\bar{\theta}_{\mathrm{eff}}}{N} \quad, \quad \bar{\theta}_{\mathrm{eff}}=\theta_{\mathrm{eff}}-2 \pi\left[\frac{\theta_{\mathrm{eff}}+\pi}{2 \pi}\right] .
$$

$f(\varphi)$ is approximately given, up to a normalization constant, by

$$
f=\exp \left\{-\sqrt{\frac{N-1}{2 N} \kappa_{0} \cos \frac{\bar{\theta}_{\mathrm{eff}}}{N}}\left(\sum_{a=1}^{N-1} \tilde{\varphi}_{a}^{2}+\sum_{a<b}^{N-1} \tilde{\varphi}_{a} \tilde{\varphi}_{b}\right)\right\} \quad, \quad \tilde{\varphi}_{a}=\varphi_{a}-\frac{\bar{\theta}_{\mathrm{eff}}}{N} .
$$



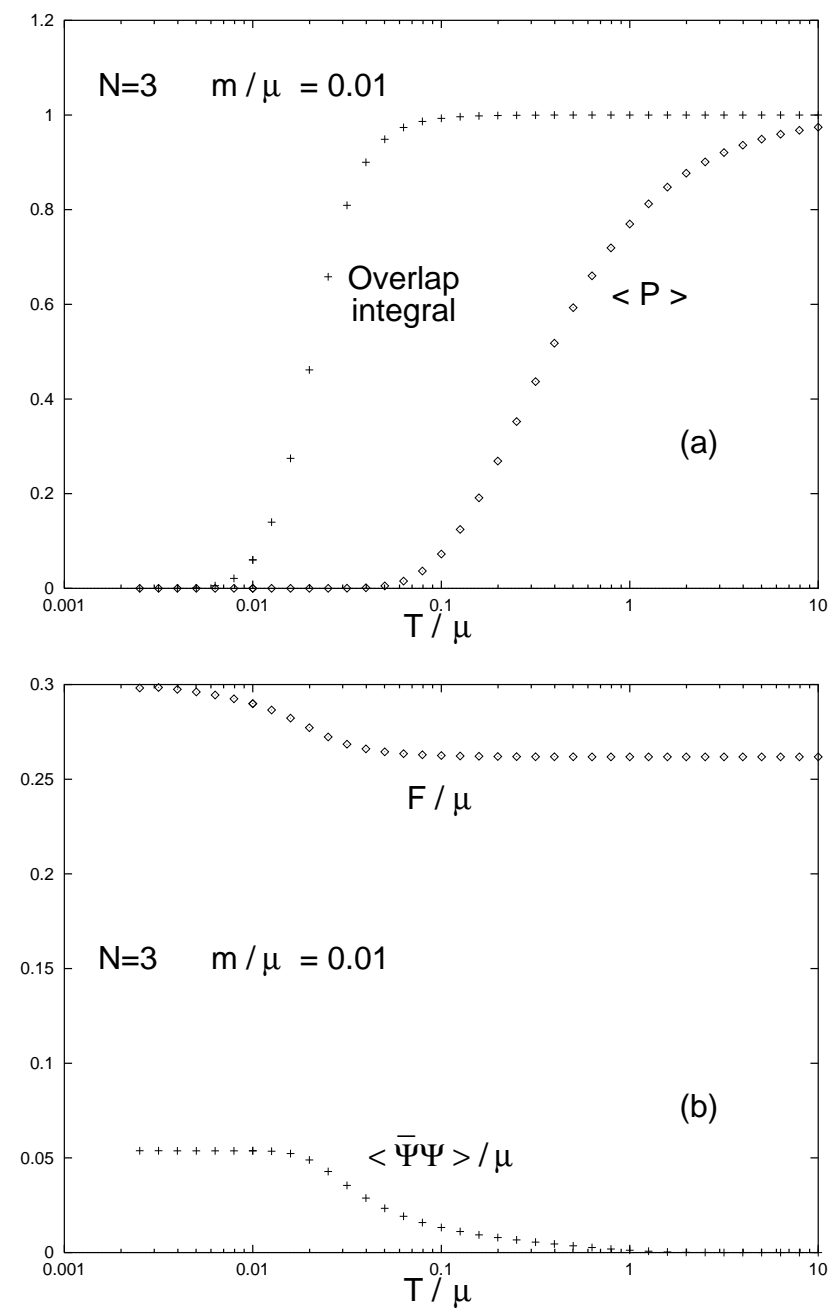

Figure 1: (a) The Polyakov loop $\langle P\rangle$ and the overlap integral in (16) are plotted as functions of $T / \mu$ for $k=1(q=e), N=3, m / \mu=0.01$ and $\theta=0$. (b) The free energy $F / \mu=-(T / \mu) \ln \langle P\rangle$ and the chiral condensate $\langle\bar{\psi} \psi\rangle / \mu$ are plotted with the same parameter values.

Hence the overlap integral gives an additional damping factor in (16). In the opposite limit $T / \mu \gg$ $1, \kappa_{0} \ll 1$ so that $f \sim$ constant. Hence we find, for an integer $k=q / e$,

$$
F_{q}(T)= \begin{cases}\frac{k^{2} \pi \mu}{4 N}\left\{1+(N-1)\left(2 e^{\gamma} \frac{m}{\mu} \cos \frac{\bar{\theta}_{\text {eff }}}{N}\right)^{\frac{N}{N+1}}\right\} & \text { for } T \ll m^{\frac{N}{N+1}} \mu^{\frac{1}{N+1}} \\ \frac{k^{2} \pi \mu}{4 N} & \text { for } T \gg \mu .\end{cases}
$$

The free energy is finite. It does not diverge even at $T=0$. An integer external charge is screened.

In fig. 1 we have depicted the temperature dependence of the Polyakov loop, free energy, and chiral condensate in the $N=3$ case with $m / \mu=0.01$ and $\theta_{\text {eff }}=0$. Notice that all these quantities show cross-over transitions, but at different temperature.

The vanishing of the Polyakov loop for a fractional charge $q=k e$ does not necessarily implies the confinement as it follows from the gauge invariance. To obtain information on the confinement 
or the string tension, we evaluate the Polyakov loop correlator:

$$
\tilde{G}_{q}(x)=\left\langle P_{q}(x)^{\dagger} P_{q}(0)\right\rangle_{\theta, T} \Longleftrightarrow G_{q=k e}(t)=\left\langle T\left[e^{-i k \Theta_{\mathrm{W}}(t)} e^{i k \Theta_{\mathrm{W}}(0)}\right]\right\rangle_{\theta, L} .
$$

Without loss of generality we suppose that $x>0$ and $t>0(x \leftrightarrow i t)$. Note that $G_{q}(t)$ is gauge invariant.

In the $N=1$ model the correlator $G_{e}(t)$ was first evaluated by Hetrick and Hosotani [24]. For a general $q, \tilde{G}_{q}(x)$ has been evaluated by Hansson et al. [12] and by Grignani et al. [15]. Consider

$$
G(t ; k, l)=\left\langle T\left[e^{-i k \Theta_{\mathrm{W}}(t)} e^{+i l \Theta_{\mathrm{W}}(0)}\right]\right\rangle_{\theta, L}
$$

in the massless $N$-flavor model, which we denote by $G^{(0)}(t ; k, l)$. In this case the zero modes $\left(\Theta_{\mathrm{W}}, q_{a}\right)$ and oscillatory modes decouple so that the model is exactly solvable. The Heisenberg operator $\Theta_{\mathrm{W}}(t)$ can be expressed in terms of Schrödinger operators

$$
\Theta_{\mathrm{W}}(t)=\Theta_{\mathrm{W}} \cos \mu t+\frac{\pi \mu L}{N} P_{\mathrm{W}} \sin \mu t+\frac{2 \pi}{N} \sum_{a=1}^{N} p_{a}(\cos \mu t-1) .
$$

Making use of (23), we find

$$
\begin{aligned}
& e^{-i k \Theta_{\mathrm{W}}(t)} e^{+i l \Theta_{\mathrm{W}}(0)}=\exp \left\{-\frac{i k l \pi \mu L}{2 N} \sin \mu t+\frac{i k \pi \mu L}{2 N} \sin \mu t(k \cos \mu t-l)\right\} \\
& \quad \times \exp \left\{-\frac{2 i k \pi}{N}(\cos \mu t-1) \sum p_{a}-i(k \cos \mu t-l) \Theta_{\mathrm{W}}\right\} \\
& \quad \times \exp \left\{-\frac{i k \pi \mu L}{N} \sin \mu t P_{\mathrm{W}}\right\} .
\end{aligned}
$$

In taking the vacuum expectation value of (24), we encounter the factor $\delta_{2 \pi}\left[\theta-\theta^{\prime}+2 \pi(k-l)\right]$ as in (15). It follows that

$$
G^{(0)}(t ; k, l)= \begin{cases}0 & \text { for } k-l \neq \text { an integer } \\ \exp \left\{-\frac{\pi \mu L}{4 N}\left(k^{2}+l^{2}-2 k l e^{-i \mu|t|}\right)\right\} & \text { for } k-l=\text { an integer. }\end{cases}
$$

This implies that the increase in the free energy in the presence of a pair of charges $q$ and $-q$ is

$$
F_{q}^{\text {pair }}(T)^{(0)}=-T \ln \left\langle P_{q}(x)^{\dagger} P_{q}(0)\right\rangle_{\theta}=\frac{\pi \mu}{2 N}\left(\frac{q}{e}\right)^{2}\left(1-e^{-\mu|x|}\right) .
$$

In the massless theory external charges are completely shielded and the string tension vanishes as shown by various authors 四, 12, 14, 15].

If fermions are massive, the situation qualitatively changes. In the literature only the $N=1$ case has been analysed for which a perturbation theory in fermion masses is valid. Restricting ourselves to $N=1$ with $k=l$ and $\delta=0$ (therefore $\theta_{\text {eff }}=\theta$ ), we find

$$
\begin{aligned}
G^{(1)}(t ; k, k) & =G(t ; k, k)-G^{(0)}(t ; k, k) \\
& =-i \int_{-\infty}^{\infty} d s\left\{\left\langle T\left[e^{-i k \Theta_{\mathrm{W}}(t)^{I}} e^{i l \Theta_{\mathrm{W}}(0)^{I}} H_{\mathrm{int}}(s)^{I}\right]\right\rangle-G^{(0)}(t ; k, k)\left\langle H_{\mathrm{int}}(s)^{I}\right\rangle\right\}
\end{aligned}
$$




$$
H_{\mathrm{int}}(s)^{I}=-\frac{m B(\mu L)}{L} \int_{0}^{L} d x\left\{e^{i q(s)^{I}} N_{\mu}\left[e^{i \sqrt{4 \pi} \phi(s, x)^{I}}\right]+\text { h.c. }\right\} .
$$

Here the superscript $I$ indicates the interaction picture defined by a massless fermion. To $\mathrm{O}(m)$ the $\phi$ field part of $H_{\text {int }}$ does not contribute.

In the second term in the expression of $G^{(1)},\left\langle H_{\text {int }}(s)\right\rangle=m B e^{-\pi / \mu L} \cos \theta$. In evaluating the first term we need, in addition to (23),

$$
q(t)^{I}=q+\frac{2}{\mu L}\left(\Theta_{\mathrm{W}}+2 \pi p\right) \sin \mu t+2 \pi P_{\mathrm{W}}(1-\cos \mu t) .
$$

A useful identity is

$$
\left\langle e^{ \pm i q(s)^{I}} e^{-i k \Theta_{\mathrm{W}}(t)^{I}} e^{i k \Theta_{\mathrm{W}}(0)^{I}}\right\rangle_{\theta}=G^{(0)}(t ; k, k) \cdot e^{\mp i \theta} e^{-\pi / \mu L} e^{\mp i \pi k\left(1-e^{i \mu t}\right) e^{-i \mu s}} .
$$

Without loss of generality we take $t>0$. The integral over $s$ in (27) splits into three parts: $\int_{-\infty}^{0}, \int_{0}^{t}$, and $\int_{t}^{\infty}$. It is easy to check that the first integral is the same as the third integral after a change of variables, and each of them vanishes. The manipulation is justified with the hypothesis of adiabatic switching of interactions implicit in the derivation of Gell-Mann-Low relations.

The second integral gives the sole contribution to $G^{(1)}$ :

$$
\begin{aligned}
& G^{(1)}(t ; k, k)=i m B e^{-\pi / \mu L} G^{(0)}(t ; k, k) \\
& \quad \times \int_{0}^{t} d s\left\{e^{-i \theta}\left(e^{2 \pi i k} e^{-i \pi k\left(e^{-i \mu(t-s)}+e^{-i \mu s}\right)}-1\right)+(\theta \rightarrow-\theta, k \rightarrow-k)\right\} .
\end{aligned}
$$

The integral is expressed in terms of Bessel functions. The correction to the free energy is, after making a Wick rotation $i t=x>0$,

$$
\begin{aligned}
F_{q}^{\text {pair }}(x, T)^{(1)}= & -T G^{(1)} / G^{(0)} \\
= & -2|x| m T B e^{-\pi / \mu L}\left\{J_{0}(2 \pi k z) \cos (\theta-2 \pi k)-\cos \theta\right. \\
& \left.+\frac{1}{\mu|x|} \sum_{n=1}^{\infty}\left(e^{-i(\theta-2 \pi k)}+(-1)^{n} e^{+i(\theta-2 \pi k)}\right) \frac{i^{n}}{n}\left(z^{-n}-z^{n}\right) J_{n}(2 \pi k z)\right\}
\end{aligned}
$$

where $z=e^{-\mu|x| / 2}$. For $\mu|x| \gg 1, z \ll 1$ so that

$$
\begin{aligned}
& F_{q}^{\text {pair }}(x, T)^{(1)} \sim \sigma|x| \\
& \sigma^{N=1}=-2 m T B\left(\frac{\mu}{T}\right) e^{-\pi T / \mu}\left\{\cos \left(\theta-\frac{2 \pi q}{e}\right)-\cos \theta\right\} .
\end{aligned}
$$

Here $\sigma$ is a "string tension". Since $\langle\bar{\psi} \psi\rangle_{\theta}=-2 T e^{-\pi T / \mu} B(\mu / T) \cos \theta$, we find

$$
\sigma^{N=1}=m\left\{\langle\bar{\psi} \psi\rangle_{\theta-2 \pi(q / e)}-\langle\bar{\psi} \psi\rangle_{\theta}\right\} .
$$

In other words, the major effect of a pair of external sources of charges $q$ and $-q$ is to shift the $\theta$ parameter in the region bounded by the sources by an amount $2 \pi(q / e)$, which changes the chiral 
condensate [4, 12]. A linear potential results because of this. We shall show below that this is true even for $N \geq 2$. The expression (33) is valid at arbitrary temperature.

The string tension $\sigma$ can be either positive or negative, depending on the values of $\theta$ and $q / e$. This implies that the $\theta \neq 0$ vacuum is unstable against pair creation of sufficiently small fractional charges.

The perturbation theory in fermion masses cannot be employed in the $N \geq 2$ case as physical quantities are not analytic in $m$ at $T=0$ [5, 13]. The perturbation theory can be applied only in the high temperature regime.

There is a better way to explore the problem. We place external charges on a circle and solve the Hamiltonian as was done in the $N=1$ case in [24] and [25].

In the presence of external charges $\mathcal{L}_{\text {ext }}=-A_{0} \rho_{\text {ext }}$. Gauss's law implies

$$
\partial_{x} E_{\text {ext }}(x)=\rho_{\text {ext }}(x)
$$

Let us restrict ourselves to static sources $\rho_{\text {ext }}(x)$ where $\int_{0}^{L} d x \rho_{\text {ext }}=0$. Then $E_{\text {ext }}(x)=E_{\text {ext }}^{(0)}-$ $A_{0}^{\text {ext }}(x)^{\prime}$. Here $E_{\text {ext }}^{(0)}$ is constant and $A_{0}^{\text {ext }}(x)=-\int_{0}^{L} d y G(x-y) \rho_{\text {ext }}(y)$. In particular, for a pair of sources located at $x=0$ and at $x=d$,

$$
\begin{aligned}
\rho_{\mathrm{ext}}(x) & =q\left\{\delta_{L}(x)-\delta_{L}(x-d)\right\} \\
E_{\mathrm{ext}}(x) & =E_{\mathrm{ext}}^{(0)}+E_{\mathrm{ext}}(x)^{(1)} \\
E_{\mathrm{ext}}^{(1)} & =-A_{0}^{\mathrm{ext}}(x)^{\prime}= \begin{cases}q\left(1-\frac{d}{L}\right) & \text { for } 0<x<d \\
-q \frac{d}{L} & \text { for } d<x<L .\end{cases}
\end{aligned}
$$

Note that $\int_{0}^{L} d x E_{\text {ext }}(x)^{(1)}=0$.

Suppose that $m_{a}=m \ll \mu$. The total charge density is $j_{\mathrm{EM}}^{0}=\sum_{a=1}^{N} e \psi_{a}^{\dagger} \psi_{a}+\rho_{\text {ext }}$, and the Coulomb energy becomes

$$
\begin{aligned}
H_{\text {Coulomb }} & =-\frac{1}{2} \int_{0}^{L} d x d y j_{\mathrm{EM}}^{0}(t, x) G(x-y) j_{\mathrm{EM}}^{0}(t, y) \\
& =\int_{0}^{L} d x \frac{1}{2}\left(\mu \chi_{1}-E_{\mathrm{ext}}^{(1)}\right)^{2}
\end{aligned}
$$

Here $\chi_{1}=N^{-1 / 2} \sum_{a=1}^{N} \phi_{a}$. In view of $(36)$ we write

$$
\begin{aligned}
& \chi_{1}=\chi_{1}^{c l}+\tilde{\chi}_{1} \\
& \left(-\frac{d^{2}}{d x^{2}}+\mu^{2}\right) \chi_{1}^{c l}=\mu E_{\mathrm{ext}}^{(1)} .
\end{aligned}
$$

The total Hamiltonian is now

$$
H_{\mathrm{tot}}^{\text {new }}=H_{0}+H_{c l}+H_{\chi}+H_{\text {mass }}
$$




$$
\begin{aligned}
& H_{c l}=\int_{0}^{L} d x \frac{1}{2}\left\{\left(\chi_{1}^{c l \prime \prime}\right)^{2}+\left(\mu \chi_{1}^{c l}-E_{\mathrm{ext}}^{(1)}\right)^{2}\right\} \\
& H_{\chi}=\int_{0}^{L} d x \frac{1}{2}:\left\{\Pi_{1}^{2}+\left(\tilde{\chi}_{1}^{\prime}\right)^{2}+\mu^{2} \tilde{\chi}_{1}^{2}+\sum_{\alpha=2}^{N}\left(\Pi_{\alpha}^{2}+\chi_{\alpha}^{\prime 2}\right)\right\}:
\end{aligned}
$$

$H_{0}$ and $H_{\text {mass }}$ are given by (6). When $m \ll \mu, \mu_{1} \sim \mu$. In $H_{\text {mass }}$

$$
N_{\mu}\left[e^{i \sqrt{4 \pi / N} \chi_{1}}\right]=e^{i \sqrt{4 \pi / N} \chi_{1}^{c l}} N_{\mu}\left[e^{i \sqrt{4 \pi / N} \tilde{\chi}_{1}}\right] .
$$

In other words, the net effect of a pair of external sources is to give $x$-dependent fermion mass phases $\delta_{a}=\sqrt{4 \pi / N} \chi_{1}^{c l}(x)$ in (西). Suppose that $\mu^{-1} \ll d \ll L$. Sufficiently away from the source

$$
\begin{aligned}
\delta_{a}^{\mathrm{eff}} & =\sqrt{\frac{4 \pi}{N}} \chi_{1}^{c l}(x) \sim \frac{2 \pi}{N e} E_{\mathrm{ext}}(x)^{(1)} \\
& = \begin{cases}\frac{2 \pi q}{N e}\left(1-\frac{d}{L}\right) \equiv \delta^{\text {in }} & \text { for } \mu^{-1}<x<d-\mu^{-1} \\
-\frac{2 \pi q}{N e} \cdot \frac{d}{L} \equiv \delta^{\text {out }} & \text { for } d+\mu^{-1}<x<L-\mu^{-1}\end{cases}
\end{aligned}
$$

Finding the exact form of the ground state wave function of (38) is rather involved. Instead, we content ourselves with finding an approximate wave function, noticing that $\delta_{a}^{\text {eff }}$ is almost constant between the two sources.

The entire circle is divided into the two regions, the inside region $0<x<d$ and outside region $d<x<L\left(\mu^{-1} \ll d \ll L\right)$. For the evaluation of local physical quantities in each region, one can approximately write the ground state as a direct product of ground states in the two regions: $\left|\Psi_{g}\right\rangle \sim|\Psi\rangle_{\text {in }} \otimes|\Psi\rangle_{\text {out }}$. In the absence of sources, $\left|\Psi_{g}\right\rangle \sim|\theta\rangle_{\text {in }} \otimes|\theta\rangle_{\text {out }}$. In the presence of sources

$$
\left|\Psi_{g}\right\rangle \sim\left|\theta+\delta \theta ; \delta^{\text {in }}\right\rangle_{\text {in }} \otimes\left|\theta+\delta \theta ; \delta^{\text {out }}\right\rangle_{\text {out }}
$$

In addition to the effect of $\delta_{a}^{\text {eff }}$ an overall shift $\delta \theta$ in the $\theta$ value results as $\delta_{a}^{\text {eff }}$ is $x$-dependent. After all there is only one $\theta$ parameter globally.

To determine $\delta \theta$, we utilize the fact that local physical quantities in the infinite volume limit $L \rightarrow \infty$ must reproduce results in the Minkowski spacetime. In particular, physics in the outside region $d+\mu^{-1}<x<L-\mu^{-1}(L \rightarrow \infty)$ must be essentially the same as physics in the absence of sources. In other words $\left|\theta+\delta \theta ; \delta^{\text {out }}\right\rangle_{\text {out }} \sim\left|\theta ; \delta^{\text {out }}=0\right\rangle_{\text {out }}$. As shown above physics depends on $\theta$ through the combination $\theta_{\text {eff }}=\theta-\sum_{a=1}^{N} \delta_{a}$. This determines

$$
\delta \theta=N \delta^{\text {out }}=-\frac{2 \pi q}{e} \cdot \frac{d}{L} .
$$

Hence, the net effect is summarized by

$$
\begin{aligned}
& \left|\Psi_{g}\right\rangle \sim\left|\theta_{\text {eff }}\right\rangle_{\text {in }} \otimes|\theta\rangle_{\text {out }} \\
& \theta_{\text {eff }}=\theta-\frac{2 \pi q}{e} .
\end{aligned}
$$


Consequently, the change in the energy due to the external sources is, to $\mathrm{O}(d / L)$,

$$
\Delta E=N m d\left\{\langle\bar{\psi} \psi\rangle_{\theta_{\mathrm{eff}}}-\langle\bar{\psi} \psi\rangle_{\theta}\right\}
$$

so that the string tension is

$$
\sigma=N m\left\{\langle\bar{\psi} \psi\rangle_{\theta-2 \pi(q / e)}-\langle\bar{\psi} \psi\rangle_{\theta}\right\}
$$

The result generalizes to finite temperature, as was seen above in the $N=1$ case.

Note that the parameter $\theta$ is not completely equivalent to the electric field $E$. Indeed, in the absence of sources $\langle E\rangle_{\theta}=\left\langle e P_{W}-A_{0}^{\prime}\right\rangle_{\theta}=0$. External sources, or external electric fields, induce effective fermion mass phases $\delta_{a}^{\text {eff }}$ in the Hamiltonian, which in turn changes the effective $\theta_{\text {eff }}$ through the chiral anomaly. We also remark that the Coulomb energy $(36)$ is $\mathrm{O}\left[(d / L)^{0}\right]$. The linear potential results from the change in the chiral condensate.

The chiral condensate $\langle\bar{\psi} \psi\rangle_{\theta}$ at arbitrary temperatue $T=L^{-1}$ has been evaluated in ref. [13]. With given $m$, the dependence of $\sigma$ on charge $q$ is essentially the $\theta$ dependence of $\langle\bar{\psi} \psi\rangle_{\theta}$. At $T=0$ it has a cusp at $\theta_{\text {eff }}=\pi(\bmod 2 \pi)$. More explicitly

$$
\sigma_{\theta}^{T=0}=-\mu^{2} \frac{N}{2 \pi}\left(2 e^{\gamma} \frac{m}{\mu}\right)^{\frac{2 N}{N+1}}\left\{\left(\cos \frac{\bar{\theta}_{\text {eff }}}{N}\right)^{\frac{2 N}{N+1}}-\left(\cos \frac{\bar{\theta}}{N}\right)^{\frac{2 N}{N+1}}\right\} .
$$

Notice the singular dependence of $\sigma$ on $m$ as well. A mass perturbation theory cannot be employed at low temperature for $N \geq 2$.

In the high temperature limit

$$
\langle\bar{\psi} \psi\rangle_{\theta}=-\frac{2 N}{\pi(N-1)} m \begin{cases}\left(\frac{\mu e^{\gamma}}{4 \pi T}\right)^{2 / N} & \text { for } m^{\frac{N}{N+1}} \mu^{\frac{1}{N+1}} \ll T \ll \mu \\ e^{-2 \pi T / N \mu} & \text { for } T \gg \mu\end{cases}
$$

for $N \geq 3$. There appears no $\theta$ dependence to this order. Hence the string tension $\sigma$ is at most $\mathrm{O}\left(\mathrm{m}^{3}\right)$ in this regime.

For $N=2$, the expressions for $\langle\bar{\psi} \psi\rangle_{\theta}$ in (47) is multiplied by a factor $2 \cos ^{2} \frac{1}{2} \theta$. Therefore

$$
\sigma_{\theta}^{N=2}=-\frac{4}{\pi} m^{2}\left(\cos \theta_{\text {eff }}-\cos \theta\right) \times \begin{cases}\frac{\mu e^{\gamma}}{4 \pi T} & \text { for } m^{\frac{2}{3}} \mu^{\frac{1}{3}} \ll T \ll \mu \\ e^{-\pi T / \mu} & \text { for } T \gg \mu\end{cases}
$$

For a general value of $T / \mu, \sigma$ must be evaluated numerically. In fig. 2 we have displayed $q / e$ dependence of $\sigma / \mu^{2}$ at $T / \mu=0.003,0.01,0.03$, and 0.1 with $m / \mu=0.01$ and $\theta=0$ in the $N=3$ case. One can see how a cusp behavior develops at $q / e=0.5\left(\theta_{\text {eff }}=\pi\right)$ as the temperature goes down. At higher temperature the magnitude of the string tension rapidly diminishes. For instance, $\sigma / \mu^{2}=1.8 \times 10^{-7}$ at $T / \mu=1$ and $q / e=0.5$.

In this paper we have shown that the confinement of fractional charges in the massive $N$ flavor Schwinger model results from the effective change in the $\theta$ parameter which alters chiral 


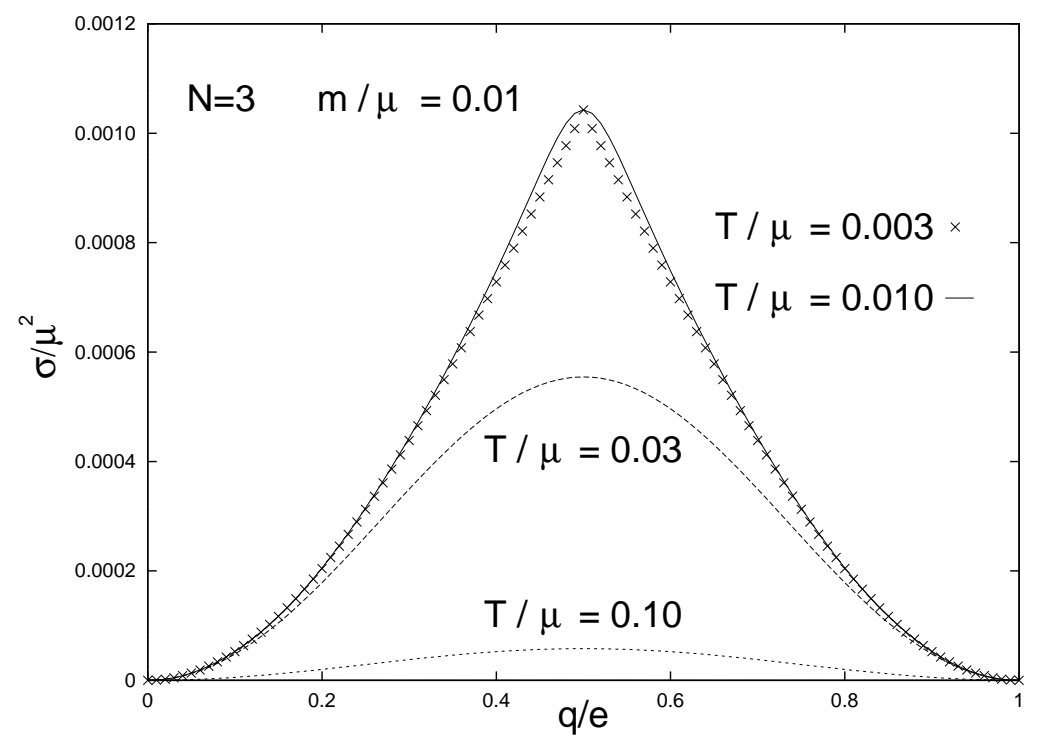

Figure 2: String tension $\sigma / \mu^{2}$ in (45) is depicted as a function of $q / e$ at various temperature in the $N=3$ case with $m / \mu=0.01$ and $\theta=0$. A cusp develops at $q / e=0.5$ in the $T \rightarrow 0$ limit.

condensates. In the multi-flavor case $(N \geq 2)$ the string tension at zero temperature has singular dependence on fermion masses and the $\theta$-parameter.

\section{Acknowledgments}

This work was supported in part by by the U.S. Department of Energy under contracts DEFG02-87ER-40328 (R.R.) and by DE-AC02-83ER-40105 (Y.H.). Y.H. would like to thank Jim Hetrick and Satoshi Iso for useful communications.

\section{References}

[1] J. Schwinger, Phys. Rev. 125 (1962) 397 ; 128 (1962) 2425.

[2] J.H. Lowenstein and J.A. Swieca, Ann. Phys. (N.Y.) 68 (1971) 172.

[3] A. Casher, J. Kogut and L. Susskind, Phys. Rev. Lett. 31 (1973) 792 ; Phys. Rev. D10 (1974) 732 .

[4] S. Coleman, R. Jackiw, and L. Susskind, Ann. Phys. (N.Y.) 93 (1975) 267.

[5] S. Coleman, Ann. Phys. (N.Y.) 101 (1976) 239.

[6] N.K. Nielsen and B. Schroer, Nucl. Phys. B120 (1977) 62 .

[7] G. Morchio, D. Pierotti and F. Strocchi, Ann. Phys. (N.Y.) 188 (1988) 217.

[8] J. Ellis, Y. Frishman, A. Hanany, M. Karliner, Nucl. Phys. B382 (1992) 189 .

[9] A.V. Smilga, Phys. Lett. B278 (1992) 371 ; Phys. Rev. D46 (1992) 5598 ; Phys. Rev. D49 (1994) 5480 ; Ann. Phys. (N.Y.) 234 (1994) 1.

[10] C. Gattringer and E. Seiler, Ann. Phys. (N.Y.) 233 (1994) 97.

[11] C. Gattringer, hep-th/9503137, hep-th/950592, MPI-PH-95-52.

[12] T.H. Hansson, H.B. Nielsen and I. Zahed, Nucl. Phys. B451 (1995) 162 . 
[13] J.E. Hetrick, Y. Hosotani and S. Iso, Phys. Lett. B350 (1995) 92 , hep-th/9510090 (to appear in Phys. Rev. D); Y. Hosotani hep-ph/9510387.

[14] D.J. Gross, I.R. Klebanov, A.V. Matytsin, A.V. Smilga, hep-th/9511104.

[15] G. Grignani, G. Semenoff, P. Sodano, O. Tirkkonen, hep-th/9511110.

[16] A. Smilga and J.J.M. Verbaarschot, hep-ph/9511471.

[17] M.B. Halpern, Phys. Rev. D13 (1976) 337.

[18] I. Affleck, Nucl. Phys. B265 [FS15] (1986) 448.

[19] A.M. Polyakov, Phys. Lett. B72 (1978) 477.

[20] L. Susskind, Phys. Rev. D20 (1979) 2610 .

[21] A. Actor, Ann. Phys. (N.Y.) 159 (1984) 445.

[22] B. Svetitsky, Phys. Report 132 (1986) 1.

[23] G. Grignani, G. Semenoff, P. Sodano, hep-th/9503109, hep-th/9504105

[24] J.E. Hetrick and Y. Hosotani, Phys. Rev. D38 (1988) 2621.

[25] S. Iso and H. Murayama, Prog. Theoret. Phys. 84 (1990) 142.

[26] D. Wolf and J. Zittartz, Z. Phys. B59 (1985) 117.

[27] N. Manton, Ann. Phys. (N.Y.) 159 (1985) 220.

[28] R. Link, Phys. Rev. D42 (1990) 2103.

[29] F.M. Saradzhev, Phys. Lett. B278 (1992) 449 ; Int. J. Mod. Phys. A9 (1994) 3179, hep-th/9501001.

[30] C. Itoi and H. Mukaida, Mod. Phys. Lett. A7 (1992) 259 .

[31] M.B. Paranjape and R. Ross, Phys. Rev. D48 (1993) 3891.

[32] M.B. Paranjape, Phys. Rev. D48 (1993) 4946.

[33] M.A. Shifman and A.V. Smilga, Phys. Rev. D50 (1994) 7659.

[34] K. Itakura and K. Ohta, Phys. Rev. D50 (1994) 4145.

[35] A.O. Barut and F.M. Saradzhev, Ann. Phys. (N.Y.) 235 (1994) 220 .

[36] S. Love, Phys. Rev. D23 (1981) 420.

[37] K. Stam and J. Visser, J. Phys. G11 (1985) L:143 .

[38] F. Ruiz Ruiz and R.F. Alvarez-Estrada, Phys. Lett. B180 (1986) 153.

[39] A. Das and A. Karev, Phys. Rev. D36 (1987) 623.

[40] G. Thompson and R.B. Zhang, J. Phys. G13 (1987) L:93 .

[41] R. Baier and E. Pilon, Z. Phys. C52 (1991) 339.

[42] I. Sachs and A. Wipf, Helv. Phys. Acta. 65 (1992) 652 .

[43] Y.C. Kao, Mod. Phys. Lett. A7 (1992) 1411.

[44] A. Fayyazuddin, T.H. Hansson, M.A. Nowak, J.J.M. Verbaarschot and I. Zahed, Nucl. Phys. B425 (1994) 553 .

[45] C. Kiefer and A. Wipf, Ann. Phys. (N.Y.) 236 (1994) 241.

[46] J. Steele, A. Subramanian and I. Zahed, Nucl. Phys. B452 (1995) 545.

[47] H. Joos, Helv. Phys. Acta. 63 (1990) 670.

[48] H. Joos and S.I. Azakov, Helv. Phys. Acta. 67 (1994) 723.

[49] H. Joos, The non-perturbative structure of gauge theories with massless fermions (Dec 1995).

[50] C. Jayewardena, Helv. Phys. Acta. 61 (1988) 636. 
[51] A. Wipf and S. Dürr, ZU-TH 30/94, ETH-TH/94-36, Nucl. Phys. B443 (1995) 201.

[52] F. Ferrari, hep-th/9310024; hep-th/9310055, Nucl. Phys. B439 (1995) 692.

[53] F. Lenz, M. Thies, S. Levit and K. Yazaki, Ann. Phys. (N.Y.) 208 (1991) 1.

[54] T. Heinzl, S. Krusche, and E. Werner, Phys. Lett. B275 (1992) 410 .

[55] K. Yee, Phys. Rev. D47 (1993) 1719

[56] H. Dilger, (Dec 93) DESY 93-181 ; Nucl. Phys. B434 (1995) 321, Int. J. Mod. Phys. C6 (1995) 123.

[57] H. Dilger and H. Joos, Nucl. Phys. B(Proc. Suppl.) 34 (1994) 195.

[58] K. Harada, T. Sugiura and M. Taniguchi, Phys. Rev. D49 (1994) 4226.

[59] V.M. Belyaev, hep-lat/9412033

[60] A.C. Irving and J.C. Sexton, hep-lat/9508032

[61] I. Horvath, hep-lat/9510018.

[62] J. Hallin and P. Liljenberg, hep-th/9601136 